\title{
BCL-2 is dispensable for thrombopoiesis and platelet survival
}

\author{
MA Debrincat ${ }^{1,2}$, I Pleines ${ }^{1,2}$, M Lebois ${ }^{1}$, RM Lane $^{1}$, ML Holmes ${ }^{1}$, J Corbin ${ }^{1}$, CJ Vandenberg ${ }^{1,2}$, WS Alexander ${ }^{1,2}$, AP Ng $^{1,2}$, A Strasser ${ }^{1,2}$, \\ P Bouillet ${ }^{1,2}$, M Sola-Visner ${ }^{3}$, BT Kile ${ }^{1,2,4}$ and EC Josefsson ${ }^{*, 1,2,4}$
}

Navitoclax (ABT-263), an inhibitor of the pro-survival BCL-2 family proteins BCL-2, BCL- $X_{L}$ and BCL-W, has shown clinical efficacy in certain BCL-2-dependent haematological cancers, but causes dose-limiting thrombocytopaenia. The latter effect is caused by Navitoclax directly inducing the apoptotic death of platelets, which are dependent on BCL- $\mathrm{X}_{\mathrm{L}}$ for survival. Recently, ABT-199, a selective BCL-2 antagonist, was developed. It has shown promising anti-leukaemia activity in patients whilst sparing platelets, suggesting that the megakaryocyte lineage does not require BCL-2. In order to elucidate the role of BCL-2 in megakaryocyte and platelet survival, we generated mice with a lineage-specific deletion of $B c / 2$, alone or in combination with loss of $M c / 1$ or $B c / x$. Platelet production and platelet survival were analysed. Additionally, we made use of BH3 mimetics that selectively inhibit BCL-2 or $B C L-X_{L}$. We show that the deletion of BCL-2, on its own or in concert with MCL-1, does not affect platelet production or platelet lifespan. Thrombocytopaenia in $B c / x$-deficient mice was not affected by additional genetic loss or pharmacological inhibition of BCL-2. Thus, BCL-2 is dispensable for thrombopoiesis and platelet survival in mice.

Cell Death and Disease (2015) 6, e1721; doi:10.1038/cddis.2015.97; published online 16 April 2015

Platelets are anucleate blood cells that play essential roles in haemostasis, wound healing and a range of other processes, including inflammation and immunity. ${ }^{1}$ They are produced by megakaryocytes, large polyploid cells that develop primarily in the bone marrow, spleen and foetal liver. ${ }^{2}$ Recent work has demonstrated that the survival of megakaryocytes and platelets is governed by the BCL-2 family proteins. ${ }^{3}$ Both cell types possess a classical BAK/BAX-mediated intrinsic apoptosis pathway that must be restrained in order for them to develop and survive.

In platelets, $B C L-X_{L}$ is the critical pro-survival $B C L-2$ family member required to keep BAK and BAX in check. The first evidence of this came from Wagner et al., ${ }^{4}$ who reported severe thrombocytopaenia in mice after MMTV-Cre-mediated deletion of Bclx in the haematopoietic system, skin and various secretory tissues. It has since been shown that megakaryocyte-restricted deletion of $B c / x$ in mice reduces platelet lifespan from $\sim 5$ days to $\sim 5 \mathrm{~h}$, with a concomitant decrease in platelet counts to $\sim 2 \%$ of wild-type levels. 5,6 Pharmacological inhibition of $\mathrm{BCL}-\mathrm{X}_{\mathrm{L}}$ with the $\mathrm{BH} 3$ mimetics ABT-737 ${ }^{7}$ or Navitoclax $(\mathrm{ABT}-263)^{8}$ (which both also inhibit $\mathrm{BCL}-2$ and $\mathrm{BCL}-\mathrm{W}$ ) triggers $\mathrm{BAK} / \mathrm{BAX}$-mediated platelet apoptosis. $^{9-11}$ As a result, these drugs cause dosedependent thrombocytopaenia in mice, dogs and humans. ${ }^{9,11-14}$ Indeed, thrombocytopaenia is the doselimiting toxicity for Navitoclax. ${ }^{12-14}$ This fact provided additional impetus for the development of agents that specifically target BCL-2, beginning with ABT-199, ${ }^{15}$ a BCL-2-selective antagonist currently in clinical trials for the treatment of a range of haematological malignancies including chronic lymphocytic leukaemia, non-Hodgkin's lymphoma, follicular lymphoma, mantle cell lymphoma, multiple myeloma and acute myeloid leukaemia. ABT-199 has already shown very promising antitumour activity, with little to no impact on platelet counts. ${ }^{15,16}$ These data suggest that BCL-2 is dispensable for the development and survival of platelets.

In megakaryocytes, BCL- $X_{L}$ is also critical for survival. Although not absolutely required for their growth and maturation, $B C L-X_{L}$ is essential for megakaryocytes to proceed safely through pro-platelet formation and platelet shedding. ${ }^{5}$ In addition to $\mathrm{BCL}-\mathrm{X}_{\mathrm{L}}$, megakaryocytes also depend on the pro-survival activity of MCL-1. Conditional deletion of $\mathrm{Mcl} 1$ alone has no effect on this lineage. In contrast, combined megakaryocyte-specific loss of $\mathrm{Bcl}$ x and $\mathrm{Mcl} / 1$ results in the failure of megakaryopoiesis, systemic haemorrhage and embryonic lethality. ${ }^{5,17,18}$ These defects are rescued by deletion of Bak and Bax. ${ }^{18}$

Consistent with the genetic studies, administration of ABT-737 to $M c / 1^{P f 4 \Delta / P f 4 \Delta}$ mice, which lack MCL-1 in megakaryocytes and platelets, induces acute, fulminant BAK/BAXdependent megakaryocyte apoptosis. Given that, in addition to $\mathrm{BCL}-\mathrm{X}_{\mathrm{L}}, \mathrm{ABT}-737$ also targets $\mathrm{BCL}-2,{ }^{7}$ these data suggested that BCL-2 might also contribute to the development and survival of the megakaryocyte lineage. This is supported by recent studies demonstrating that neonatal human platelets contain increased levels of BCL-2 relative to adult counterparts, ${ }^{19}$ and that platelet lifespan is extended in transgenic mice expressing BCL-2 under the control of the

\footnotetext{
${ }^{1}$ The Walter and Eliza Hall Institute of Medical Research, 1 G Royal Parade, Parkville, VIC, Australia; ${ }^{2}$ Department of Medical Biology, The University of Melbourne, 1 G Royal Parade, Parkville, VIC, Australia and ${ }^{3}$ Boston Children's Hospital, Division of Newborn Medicine, Boston, MA, USA

*Corresponding author: EC Josefsson, Cancer \& Haematology Division, The Walter and Eliza Hall Institute of Medical Research, 1G Royal Parade, Parkville, Victoria 3052 , Australia. Tel: +61 39345 2484; Fax: +61 39345 2922; E-mail: josefsson@ @ehi.edu.au

${ }^{4}$ These authors contributed equally to this work.

Abbreviations: E, embryonic day; FL, foetal liver; H\&E, haematoxylin and eosin; TO, thiazole orange; TPO, thrombopoietin; P, perinatal day Received 12.2.15; revised 25.2.15; accepted 03.3.15; Edited by G Raschellà
} 
pan-haematopoietic Vav promoter. ${ }^{20}$ In light of these observations, and intense ongoing activity surrounding the development of novel $\mathrm{BH} 3$ mimetics, ${ }^{21}$ we set out to elucidate the role of BCL-2 in megakaryocytes and platelets. Mice with a megakaryocyte-specific deletion of $\mathrm{Bcl} 2$, either alone or in combination with deletion of $\mathrm{Mcl} 1$ or $\mathrm{Bcl}$, were generated. The effect of these mutations, and of BCL-2 or BCL- $\mathrm{X}_{\mathrm{L}}$ selective $\mathrm{BH} 3$ mimetics, on the megakaryocyte lineage was assessed.

\section{Results}

Platelet production and platelet lifespan are normal in the absence of BCL-2. Mice lacking BCL-2 in the megakaryocytic lineage were generated by crossing animals carrying a floxed allele of $\mathrm{Bcl}^{22}$ with Pf4-Cre transgenic animals. ${ }^{23}$ $B C l 2^{P f 4 \Delta / P f 4 \Delta}$ mice were born at the expected Mendelian ratios, and were outwardly healthy. Deletion of BCL-2 in bone marrow-derived megakaryocytes and washed platelets was confirmed by western blotting (Figure 1a). Peripheral blood platelet counts (Figure 1b) and platelet survival (Figure 1c) in adult $B c / 2^{P f 4 \Delta / P f 4 \Delta}$ mice were comparable with control animals. Megakaryocyte numbers and ploidy in bone marrow of $\mathrm{Bcl} 2^{P f 4 \Delta / P f 4 \Delta}$ mice were normal (Figures $1 \mathrm{~d}$ and $\mathrm{e}$ ). Additionally, we assessed platelet and megakaryocyte counts in young (1-5-week old) mice with a constitutive deletion of
Bcl2. ${ }^{24}$ Despite their various phenotypic abnormalities, including kidney polycystic disease, growth retardation and lymphopaenia, blood platelet counts (Figure 1f) and bone marrow and spleen megakaryocyte numbers (Figure 1g) in $\mathrm{Bcl}^{-/}$mice were comparable with those of wild-type controls. Together, these results indicated that BCL-2 is dispensable for steady state platelet production. To establish whether this is also the case under conditions of stress, we induced transient thrombocytopaenia by injecting anti-platelet serum. This typically leads to platelet depletion in wild-type mice within $24 \mathrm{~h}$, followed by recovery and rebound thrombocytosis at $\sim 5$ days post injection. Bcl $2^{P f 4 \Delta / P f 4 \Delta}$ and $B c / 2^{f / / f l}$ mice responded similarly to anti-platelet serum treatment (Figure $1 \mathrm{~h}$ ), indicating that even under conditions of stress thrombopoiesis, BCL-2 is dispensable for the development and survival of megakaryocytes and platelets.

Combined loss of BCL-2 and MCL-1 does not affect platelet production or platelet survival. We and others have previously shown that platelet production and platelet counts are normal in $M c / 1^{P f 4 \Delta / P f 4 \Delta}$ mice, whereas combined deletion of $\mathrm{Mcl} 1$ and $\mathrm{Bcl} x$ in megakaryocytes results in haemorrhage and embryonic lethality. ${ }^{17,18}$ To examine any potential functional redundancy between BCL-2 and MCL-1 in the megakaryocyte lineage, we conditionally deleted both of the genes encoding these proteins. We began by a

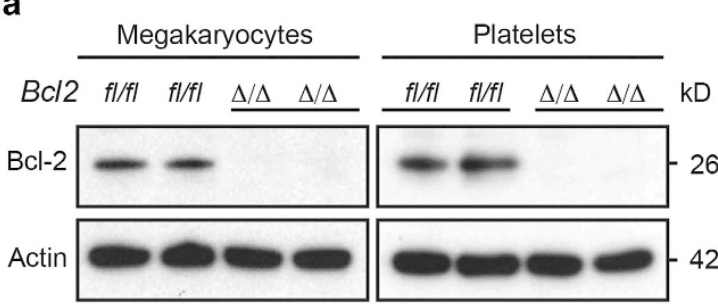

e

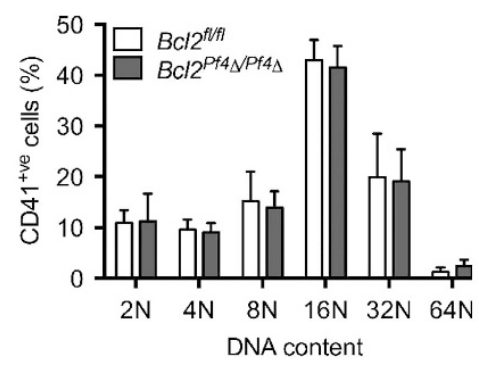

f

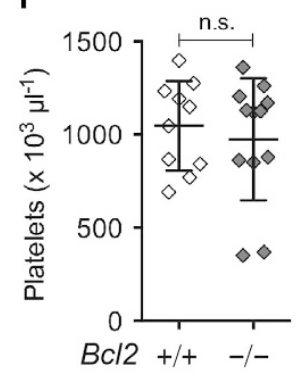

b

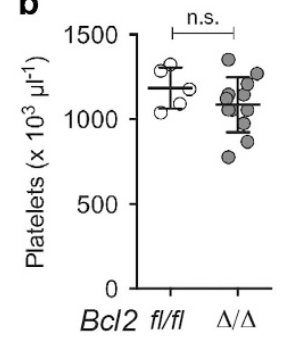

c

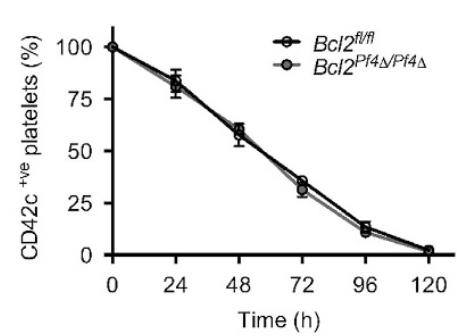

d

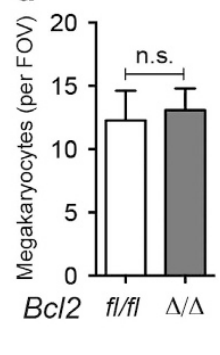

g

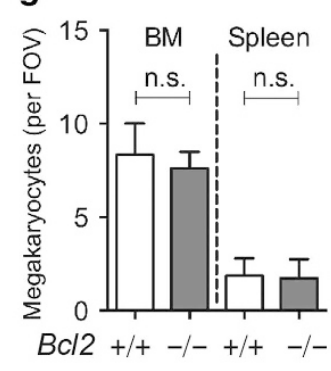

h

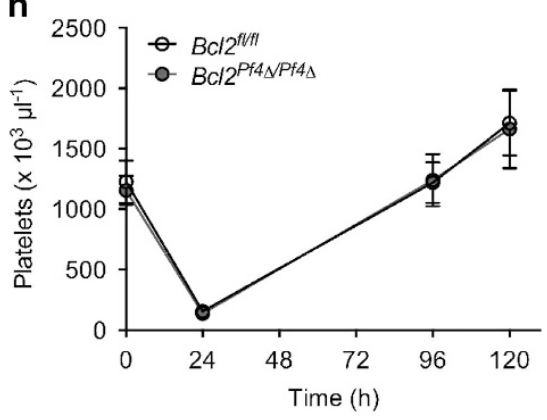

Figure 1 Loss of BCL-2 does not affect platelet survival or platelet production. (a) Western blot analysis of protein lysates from platelets and bone marrow-derived megakaryocytes from $B c / 2^{f / f / l}$ and $B c / 2^{P f 4 \Delta / P f 4 \Delta}$ mice. Bone marrow progenitor cells were cultured in thrombopoietin (TPO), and mature megakaryocytes were purified on a BSA gradient. Probing for actin was used as a control for protein loading. Each lane represents platelets from an individual mouse. (b) Platelet counts in $B c / 2^{P f 4 \Delta / P f 4 \Delta}$ and $B c / 2^{f / f t l}$ control mice at 7-10 weeks of age. Each symbol represents an individual mouse. (c) Platelet survival curves in $B c / 2^{P f 4 \Delta / P f 4 \Delta}$ and $B c / 2^{f / f t l}$ control mice. Platelets were labelled via

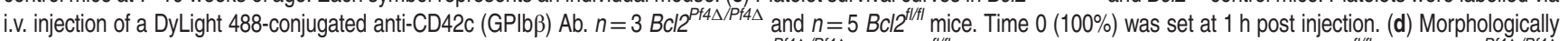

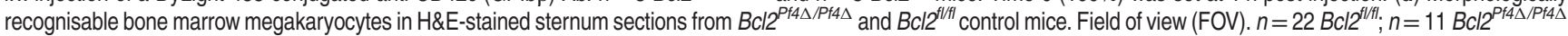

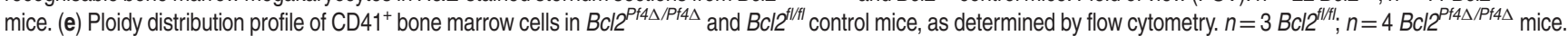
(f) Platelet counts in $B c / 2^{-/}$and $B c / 2^{+/+}$mice at 2-5 weeks of age. Each symbol represents an individual mouse. (g) Morphologically recognisable bone marrow (BM) and spleen megakaryocytes in H\&E-stained sections from $\mathrm{Bcl}^{-/-}$and $\mathrm{Bcl} / 2^{+/+}$mice at $1-4$ weeks of age. $n=5 \mathrm{Bcl2}{ }^{+/+}, n=6 \mathrm{Bcl} 2^{-/}$mice. (h) Platelet counts in mice treated with antiplatelet serum. $n=5-6 B c / 2^{f / f t}, n=4 B c / 2^{P f 4 \Delta / P f 4 \Delta}$ mice per time point (except $\left.96 h, n=2\right)$. Data are presented as mean \pm S.D. 
measuring body weight, platelet counts and the proportion of reticulated platelets in newborn mice 5-10 days after birth. $B C l 2^{P f 4 \Delta / P f 4 \Delta} M c / 1^{P f 4 \Delta / P f 4 \Delta}$ double knockout animals gained weight at a rate comparable with control littermates (Supplementary Figure 1a). Platelet counts significantly increased from day 7 to 10 in all mice with no differences between genotypic classes (Figure 2a). This correlated with a reduction in the proportion of reticulated (thiazole orangestained) platelets (Figure 2b). In adult Bcl2 ${ }^{P f 4 \Delta / P f 4 \Delta}$ $M c / 1^{P f 4 \Delta / P f 4 \Delta}$ mice, platelet counts and platelet survival were equivalent to those of control littermates (Figures $2 c$ and $d$ ). We confirmed efficient deletion of BCL-2 and MCL-1 in bone marrow-derived BSA-gradient-purified megakaryocytes by western blotting (Supplementary Figure 1b). Bone marrow megakaryocyte numbers and ploidy were normal in $B c l 2^{P f 4 \Delta / P f 4 \Delta} M c / 1^{P f 4 \Delta / P f 4 \Delta}$ mice, and these animals exhibited a wild-type response to anti-platelet serum-induced thrombocytopaenia (Figures $2 \mathrm{e}$ and $\mathrm{f}$, Supplementary Figure 1c).
Consistent with these observations, administration of a single dose of the BCL-2-selective antagonist ABT-199 $(100 \mathrm{mg} / \mathrm{kg})$ to $M c / 1^{P f 4 \Delta / P f 4 \Delta}$ and $M c / 1^{f l f l}$ mice did not markedly affect blood platelet or bone marrow megakaryocyte numbers 6 and $24 \mathrm{~h}$ post injection (Figures $2 \mathrm{~g}$ and $\mathrm{h}$ ). Although a statistically significant reduction in platelet counts at $24 \mathrm{~h}$ post treatment was observed in $M c / 1^{P f 4 \Delta / P f 4 \Delta}$ mice, relative to $M c / 1^{f / / f l}$ counterparts (Figure $2 \mathrm{~g}$ ), the numbers were in the normal range $\left(987.6 \pm 235.1 \times 10^{3} / \mu \mathrm{l}\right)$ and not significantly different from vehicle-treated $M c / 1^{P f 4 \Delta / P f 4 \Delta}$ animals. Collectively, these results demonstrated that the combined functions of $B C L-2$ and $\mathrm{MCL}-1$ are dispensable for platelet production and platelet survival.

BCL- $X_{L}$ antagonism in MCL-1/BCL-2-deficient mice. The recent development of $B C L-X_{L}$-specific $B H 3$ mimetic compounds ${ }^{25-27}$ enabled us to determine the combined effect of $B C L-X_{L}, B C L-2$ and $M C L-1$ inhibition on the
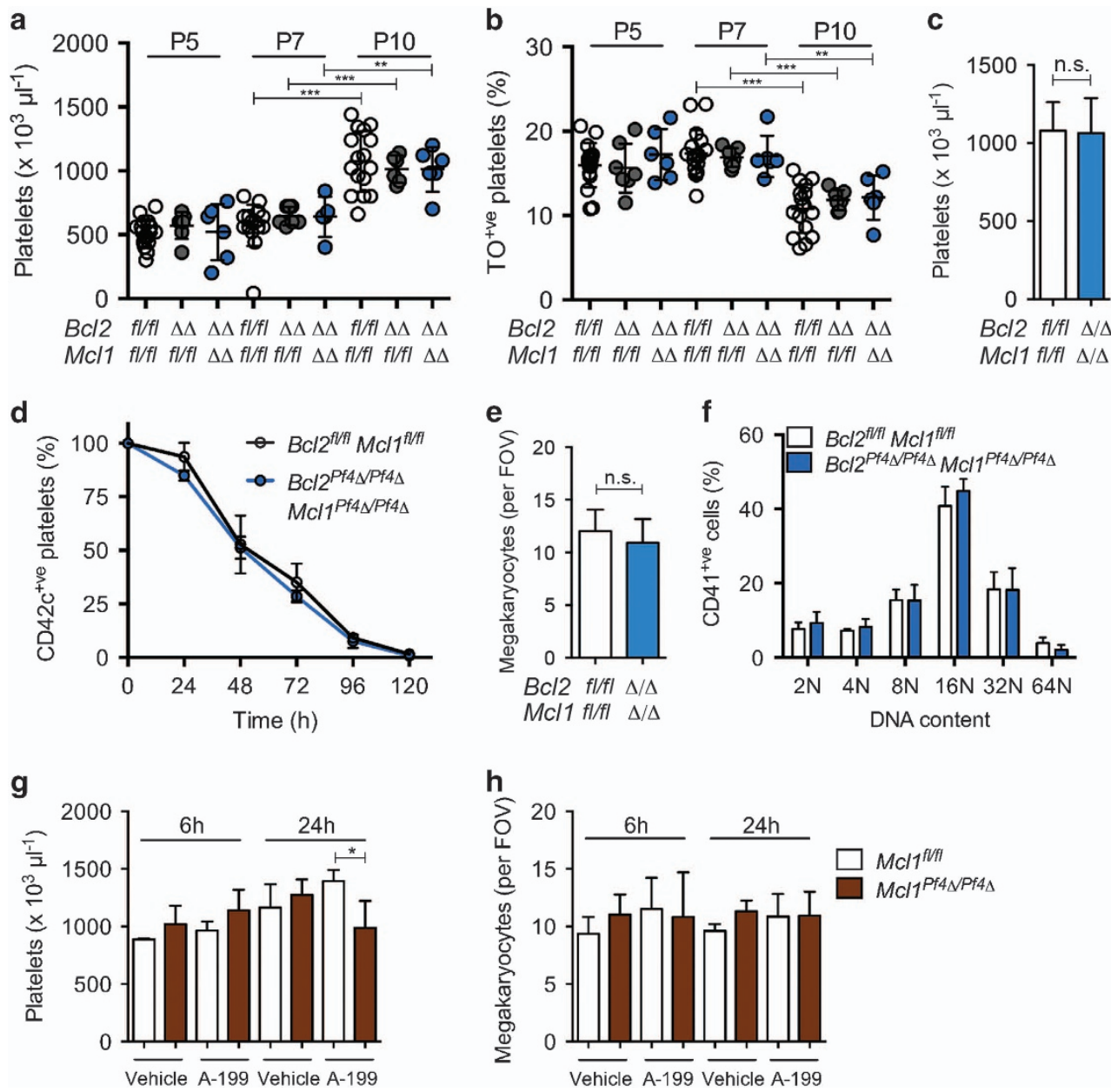

Figure 2 Combined deletion of BCL-2 and MCL-1 does not affect platelet production. (a) Platelet counts and (b) \% reticulated thiazole orange (TO)-positive platelets in floxed control, $B c / 2^{P f 4 \Delta / P f 4 \Delta}$ and $B c / 2^{P f 4 / P f 4 \Delta} M c / 1^{P f 4 \Delta / P f 4 \Delta}$ neonatal mice on perinatal day (P) 5,7 and 10 . Each symbol represents an individual mouse. (c) Platelet counts in

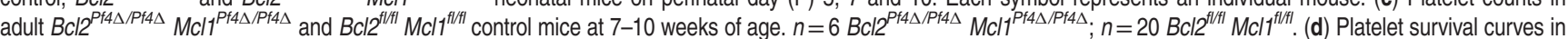
$B c 12^{P f 4 \Delta / P f 4 \Delta} M c / 1^{P f 4 \Delta / P f 4}$ and floxed control mice. Platelets were labelled via i.v. injection of a Dylight 488-conjugated anti-CD42c mAb. $n=6$ mice per genotype. Time $0(100 \%)$ was set at $8 \mathrm{~h}$ post injection. (e) Numbers of morphologically recognisable megakaryocytes in H\&E-stained bone marrow sections. $n=4 B c / 2^{f / f t l} M c / 1^{f / f t i} ; n=7 B c / 2^{P(4 \Delta / P f 4 \Delta}$

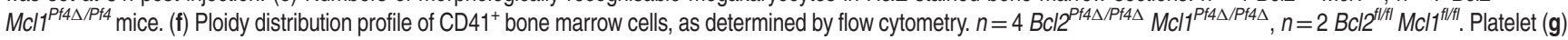
and bone marrow megakaryocyte counts (h) 6 and $24 \mathrm{~h}$ post oral administration of the BCL-2 selective BH3 mimetic ABT-199 (A-199) $100 \mathrm{mg} / \mathrm{kg}$ or vehicle control. $n=3-5$ mice per group, except $M c / 1^{t / f / t} 6 \mathrm{~h}$ vehicle where $n=2 .{ }^{*} P<0.05 ;{ }^{* \star} P<0.005 ;{ }^{* \star *} P<0.001$. Data are presented as mean \pm S.D. 
megakaryocyte lineage in vivo. Bcl2 ${ }^{P f 4 \Delta / P f 4 \Delta} M c / 1^{P f 4 \Delta / P f 4 \Delta}$ double knockout and control littermates were treated with a single dose of the BCL- $\mathrm{X}_{\mathrm{L}}$-selective antagonist A-1155463.7 $(\mathrm{A}-463,5 \mathrm{mg} / \mathrm{kg})$. In Bcl $2^{f / / f l} \mathrm{Mcl} 1^{\mathrm{fl} / f l}$ mice, A-463 induced rapid thrombocytopaenia, but had no effect on bone marrow megakaryocyte numbers at 2 and $24 \mathrm{~h}$ post injection (Figures $3 \mathrm{a}, \mathrm{b}$ and d). In contrast, in $B c / 2^{P f 4 \Delta / P f 4 \Delta} M c / 1^{P f 4 \Delta / P f 4 \Delta}$ animals, acute thrombocytopaenia was observed (Figure 3a). Additionally, apoptotic megakaryocytes with pyknotic nuclei were apparent in the bone marrow and spleen $2 \mathrm{~h}$ post administration (Figures $3 c$ and $d$ ). Megakaryocyte numbers were reduced, and consistent with this, platelet counts were

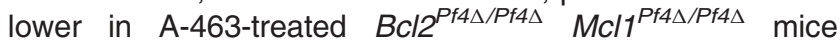
compared with floxed control animals $24 \mathrm{~h}$ post injection (Figures $3 \mathrm{a}$ and $\mathrm{b}$ ). These results prompted us to explore functional redundancy between $B C L-2$ and $B C L-X_{L}$ in the control of cell survival in megakaryocytes and platelets.

Inhibition of BCL-2 does not exacerbate thrombocytopaenia in BCL- $X_{L}$-deficient mice. We administered the BCL-2-selective antagonist ABT-199 (100 mg/kg) to $B c / x^{P f 4 \Delta / P f 4 \Delta}$ and floxed control littermates by oral gavage and measured platelet and megakaryocyte counts 6 and $24 \mathrm{~h}$ post treatment. Conditional deletion of $B C L-X_{L}$ in the megakaryocytic lineage causes a profound shortening in platelet lifespan, consequent thrombocytopaenia and reactive megakaryocytosis. ${ }^{5}$ Consistent with this, vehicle-treated $B c / x^{P f 4 \Delta / P f 4 \Delta}$ mice exhibited thrombocytopaenia and increased megakaryocyte counts (Figures $4 a$ and b). No significant exacerbation of these deficient platelet and megakaryocyte numbers were observed when $B c / x^{P f 4 \Delta / P f 4 \Delta}$ mice were treated with ABT-199 (Figures $4 a$ and b). To complement the pharmacological approach, we generated $B c l 2^{P f 4 \Delta / P f 4 \Delta} B c / x^{P f 4 \Delta / P f 4 \Delta}$ double knockout mice. Similar to their $B c / x^{P f 4 \Delta / P f 4 \Delta}$ littermates, Bcl $2^{P f 4 \Delta / P f 4 \Delta} B c / x^{P f 4 \Delta / P f 4 \Delta}$ animals were present at weaning at $\sim 75 \%$ of the number expected (25 observed/33 expected). Platelet counts in adult
$B \mathrm{cl} 2^{+/+} \quad B c / x^{P f 4 \Delta / P f 4 \Delta}$ and $B c / 2^{P f 4 \Delta / P f 4 \Delta} \quad B c l x^{P f 4 \Delta / P f 4 \Delta}$ mice were identical (Figure $4 c$ ). In $B c / x$ heterozygotes $\left(B c / x^{P f 4 \Delta / f f}\right)$, deletion of one or both alleles of $B c / 2$ had no additional impact on platelet numbers and lifespan (Figure 4d). Megakaryocyte counts in bone marrow and

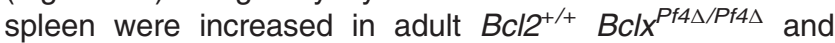
$B c l 2^{P f 4 \Delta / P f 4 \Delta} B c / x^{P f 4 \Delta / P f 4 \Delta}$ mice relative to control animals (Figure 4e). We did observe a modest, but statistically significant, decrease in bone marrow megakaryocytes, and an increase in splenic megakaryocytes in $B c / 2^{P f 4 \Delta / P f 4 \Delta}$ $B c / x^{P f 4 \Delta / P f 4 \Delta}$ animals relative to $B c / 2^{+/+} B c l x^{P f 4 \Delta / P f 4 \Delta}$ counterparts. However, these mice were bred in different colonies, which likely explains the minor differences. As expected, and similar to $B c / x^{P f 4 \Delta / P f 4 \Delta}$ mice, megakaryocyte ploidy was increased in $B c / 2^{P f 4 \Delta / P f 4 \Delta} B c / x^{P f 4 \Delta / P f 4 \Delta}$ animals (Figure 4f).

Pharmacological inhibition of $B C L-X_{L}$, but not BCL-2, induces megakaryocyte apoptosis. The results from our in vivo studies suggested that genetic or pharmacological antagonism of BCL-2 has no adverse effect on megakaryopoiesis. To test this directly in isolated primary cells, we treated foetal liver or bone marrow-derived mouse megakaryocytes with ABT-737, ABT-199 or A-463. It has been previously reported that in cultured primary mouse megakaryocytes, deletion of $B c /-x$ or treatment with $A B T-737$ triggers loss of cell viability, Caspase-3/7 activity and a failure of proplatelet formation. ${ }^{5}$ In line with these data, we found that the BCL- $X_{L}$-selective inhibitor A-463 induced dosedependent Caspase-3/7 activation in wild-type foetal liverderived megakaryocytes (Figure $5 \mathrm{a}$ ). Consistent with its increased potency against $B C L-X_{L}^{27}, A-463$ treatment at a concentration of $2.5 \mu \mathrm{M}$ induced more caspase activity in megakaryocytes than $\mathrm{ABT}-737$ at $5 \mu \mathrm{M}$. In contrast, treatment with the BCL-2 antagonist ABT-199 $(2.5 \mu \mathrm{M})$ had no effect. Combination treatment with A-463 and ABT-199 did not amplify Caspase-3/7 activity beyond that seen with A-463 treatment alone. Similarly, loss of Bcl2 did not render bone
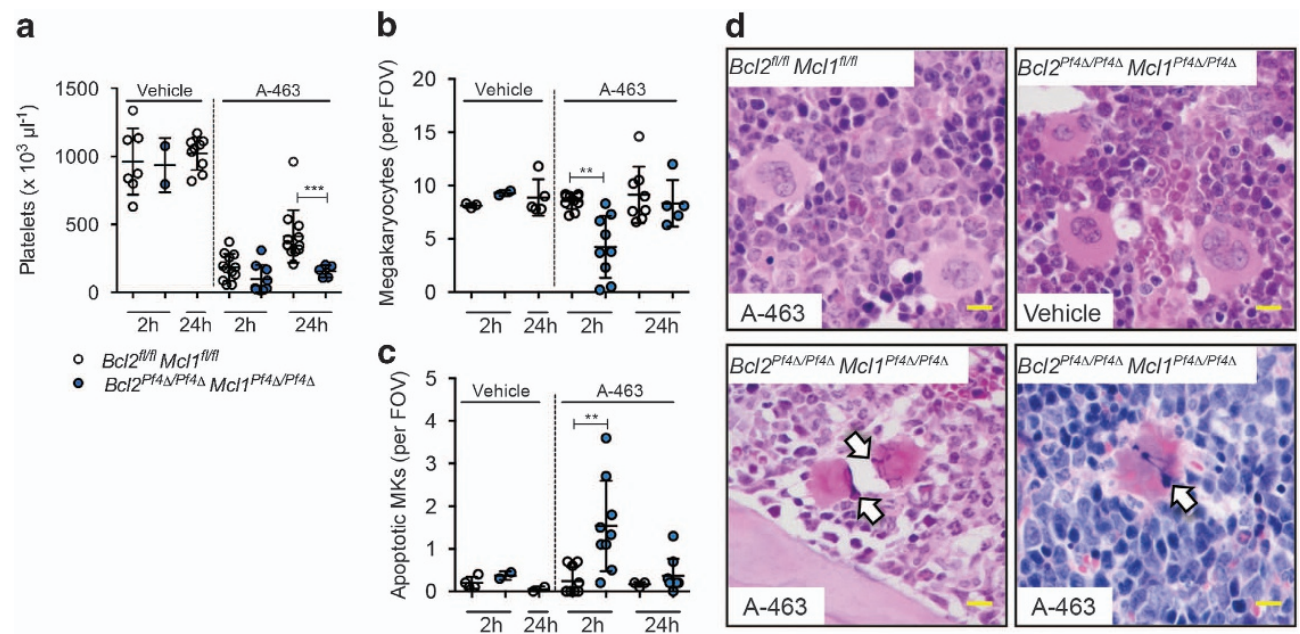

Figure $3 \mathrm{BCL}-\mathrm{X}_{\mathrm{L}}$ inhibition leads to thrombocytopaenia and apoptosis of MCL-1/BCL-2 deficient megakaryocytes in vivo. Platelet (a) and bone marrow megakaryocyte

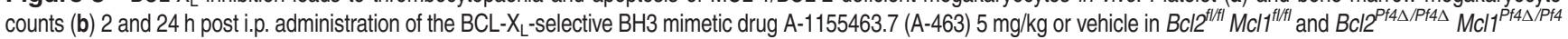
mice. Each symbol represents an individual mouse. ${ }^{* \star} P<0.005$; ${ }^{* *} P<0.001$. Data are presented as mean \pm S.D. (c) Apoptotic bone marrow megakaryocytes with pyknotic nuclei in H\&E sections. (d) Representative images of H\&E-stained spleen (lower right panel) and bone marrow (all other panels) megakaryocytes $2 \mathrm{~h}$ post A-463 or vehicle treatment. Scale bar: $20 \mu \mathrm{m}$. Pyknotic nuclei are indicated by white arrows 
a

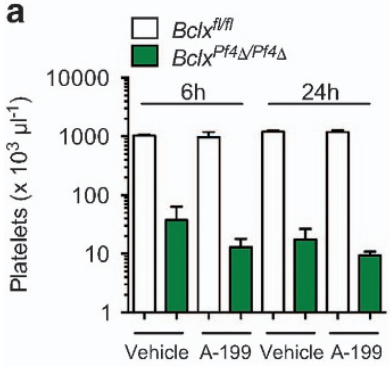

d

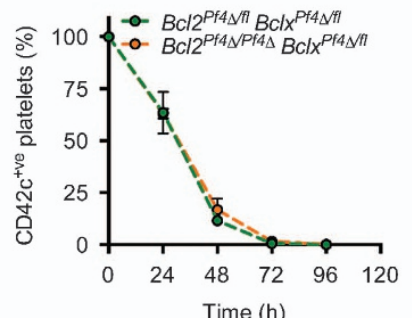

b

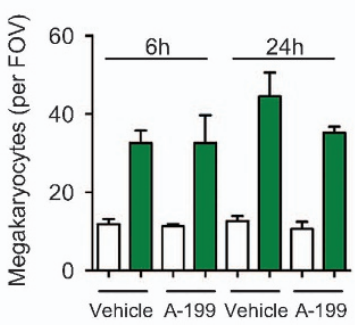

e

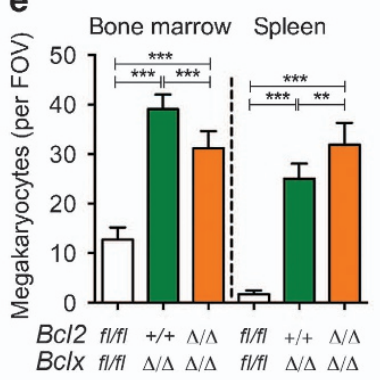

C

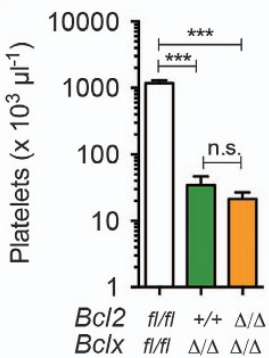

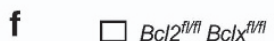

$\square B C / 2^{P f 4 \Delta P f 4 \Delta} B C / x^{P f 4 \Delta P f 4 \Delta}$

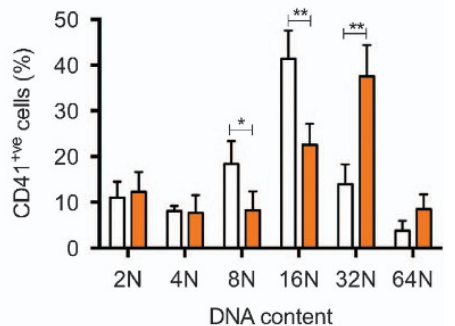

Figure 4 Thrombocytopaenia in $B c l x$-deficient mice is not exacerbated by BCL-2 inhibition. Platelet (a) and bone marrow megakaryocyte counts (b) 6 and $24 \mathrm{~h}$ post oral administration of the BCL-2-selective BH3 mimetic ABT-199 (A-199) $100 \mathrm{mg} / \mathrm{kg}$ or vehicle control. $n=3-4$ mice per group, except $B c l x^{P f 4 \Delta / P f 4 \Delta} 24 \mathrm{~h}$ vehicle where $n=2$. Platelet counts are shown on a log scale. (c) Platelet counts (log scale) in $B c / 2^{P f 4 \Delta / P f 4 \Delta} B c l x^{P f 4 \Delta / P f 4 \Delta}, B c l 2^{+/+} B c l x^{P f 4 \Delta / P f 4 \Delta}$ and floxed control mice at 7-10 weeks of age. (d) Platelet

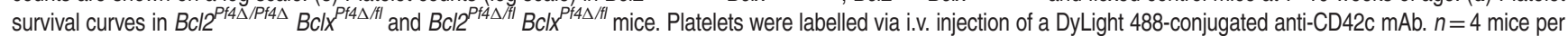
genotype. Time $0(100 \%)$ was set at $1 \mathrm{~h}$ post injection. (e) Numbers of morphologically recognisable megakaryocytes in H\&E-stained bone marrow and spleen sections. $n=8-17$ mice per genotype. (f) Ploidy distribution profile of $\mathrm{CD} 41^{+}$bone marrow cells, as determined by flow cytometry. $n=4$ mice per genotype. ${ }^{\star} P<0.05 ;{ }^{* \star} P<0.005$; ${ }^{* \star \star} P<0.001$. Data represent mean \pm S.D.

a

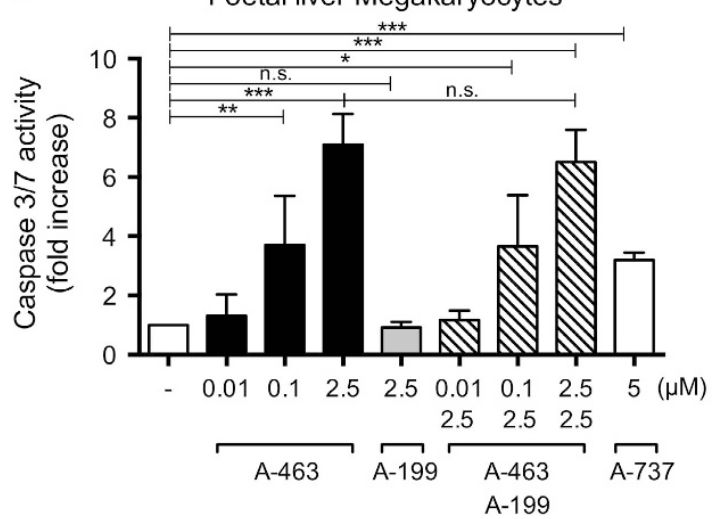

b Bone marrow Megakaryocytes

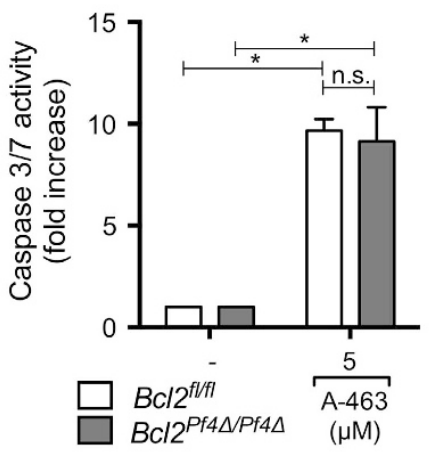

Figure $5 \mathrm{BCL}-\mathrm{X}_{\mathrm{L}}$ inhibition induces cell death in megakaryocytes in vitro. (a) Caspase-3/7 activity expressed as fold-increase in wild-type foetal liver-derived BSA gradientpurified megakaryocytes $5 \mathrm{~h}$ post treatment with A-1155463.7 (A-463) 0.01-2.5 $\mu \mathrm{M}, \mathrm{ABT}-199$ (A-199) $2.5 \mu \mathrm{M}, \mathrm{ABT}-737$ (A-737) $5 \mu \mathrm{M}$ or vehicle (DMSO) was assessed by using the Caspase-3/7 Glo (Promega) kit. $n=4-5$ biological replicates, three independent experiments. (b) Caspase-3/7 activity expressed as fold-increase in bone marrow-derived BSA gradient-purified $B C 2^{P f 4 \Delta / P f 4 \Delta}$ and $B c / 2^{f / / f l}$ megakaryocytes $5 \mathrm{~h}$ post treatment with $\mathrm{A}-463(5 \mu \mathrm{M})$ or vehicle (DMSO) was assessed by using the Caspase-3/7 Glo kit. Data shown are representative of two independent experiments. ${ }^{*} P<0.05$; ${ }^{\star \star} P<0.005 ;{ }^{\star \star \star} P<0.001$. Data represent mean \pm S.D.

marrow-derived megakaryocytes more sensitive to A-463 (Figure 5b).

\section{Discussion}

In this study, we analysed platelet production in mice with a megakaryocytic lineage-specific deletion of $\mathrm{Bcl} 2$ alone or in combination with deletion of $\mathrm{Mcl} 1 \mathrm{or} B c / x$. In addition, selective
BH3 mimetics inhibiting BCL-2 or BCL- $\mathrm{X}_{\mathrm{L}}$ were assessed. Our genetic and pharmacological studies demonstrate that BCL-2 is dispensable for platelet production both at steady state and under conditions of stress. Moreover, platelet survival in vivo was not affected by genetic loss of BCL-2 or its pharmacological inhibition. This aligns with initial reports on the effects of ABT-199 in patients, ${ }^{15,16}$ and bodes well for the clinical development of BCL-2 antagonists. Our experiments confirm 
the importance of $B C L-X_{L}$ and the ancillary role of $M C L-1$ in maintaining megakaryocyte viability and $B C L-X_{L}$ in sustaining platelet survival. $^{5}$

Our data indicate that BCL-2 is dispensable for platelet survival in adults, and in neonates as well. The latter findings are consistent with a recent study from Liu and colleagues, ${ }^{19}$ which demonstrated that although neonatal platelets display elevated BCL-2 protein levels and extended survival compared with adult platelets, BCL-2 was not the primary molecule facilitating this effect. We have further demonstrated that conditional deletion of $\mathrm{Bcl} 2$, on its own or in concert with $\mathrm{Mcl}$, had no impact on neonatal platelet counts.

$\mathrm{BCL}-2$ has recently been linked to myeloid progenitor cell survival. ${ }^{28}$ As Cre-dependent recombination utilising the Pf4Cre transgenic mouse is restricted to megakaryocytes, platelets $^{23}$ and a small fraction of late megakaryocyte progenitors, ${ }^{29}$ we could not use this model to address the role of BCL-2 in the earliest stages of megakaryopoiesis. However, mice carrying the constitutive deletion of $\mathrm{Bcl} 2$ did afford this opportunity. Germline loss of BCL-2 leads to polycystic kidney disease, lymphopaenia, grey fur (due to premature death of melanocytes), growth retardation and early mortality. ${ }^{24,30,31}$ Even with the complex co-morbidities of this model, our results show that young $\mathrm{Bcl}^{-/}$and control mice exhibited similar megakaryocyte and platelet counts, indicating that loss of BCL-2 in progenitor cells does not significantly affect the megakaryocytic lineage at steady state in young animals. Although it is increasingly clear that ABT-199 used as a single agent within the therapeutic window will not kill platelets and megakaryocytes, potential effects on progenitor cells with subsequent thrombocytopaenia may become apparent when used in combination with certain chemotherapeutics. This is pertinent as clinical trials are currently evaluating the safety and efficacy of ABT-199 in combination with proteasome inhibitors in multiple myeloma, ${ }^{32}$ hypomethylating agents in acute myeloid leukaemia, ${ }^{33}$ and alkylating or antimitotic drugs in non-Hodgkin's lymphoma, chronic lymphocytic leukaemia and follicular lymphoma ${ }^{34,35}$ known to cause thrombocytopaenia through bone marrow suppression or inhibition of proplatelet formation. ${ }^{36,37}$

Despite dose-limiting thrombocytopaenia, BCL- $\mathrm{X}_{\mathrm{L}}$ antagonism has shown encouraging results in certain solid tumours. $^{12} 38$ Combination trials with Navitoclax and kinase inhibitors (MEK, RAF and BRAF) are underway for advanced or metastatic solid tumours, including small-cell lung, colon, pancreatic, rectal and liver cancer. Limited information is available on the effects of this group of kinase inhibitors on megakaryocytes and platelets, although MEK inhibition has been associated with thrombocytopaenia. ${ }^{39}$ Hence, for combination treatments using $\mathrm{BCL}-\mathrm{X}_{\mathrm{L}}$ antagonists and kinase inhibitors, it will be imperative to closely monitor platelet counts, as both platelet production and platelet survival may be affected. With the recent development of BCL- $X_{L}-$ specific $\mathrm{BH} 3$ mimetics, ${ }^{25-27}$ there is a need to find means of sustaining platelet counts in order to allow safe dose escalation without increased risk of bleeding. ${ }^{40}$ Although $B C L-2$ is not required for platelet survival, we recently showed that overexpression of BCL-2 in blood cells extends platelet lifespan in adult mice, ${ }^{20}$ similarly to that observed in animals lacking the essential mediators of intrinsic apoptosis, BAK and BAX. ${ }^{5,9}$
One could imagine that one approach to facilitating the safe administration of $B C L-X_{L}$ antagonists might be transfusion of platelets either overexpressing BCL-2, or lacking BAK/BAX, thus rendering them resistant to $B C L-X_{L}$ inhibition. Recent advances in this field of research, including development of human-induced pluripotent stem cell-derived megakaryocytes generating platelets, ${ }^{41}$ HLA-universal platelets, ${ }^{42}$ synthetic micro-vessels ${ }^{43}$ and a novel microfluidic bioreactor design enabling ex vivo platelet production, ${ }^{44}$ might allow such manipulation in the not-too-distant future.

\section{Materials and Methods}

Mice. Bcl2 ${ }^{1-24} \mathrm{Bcl} / 2$ floxed, ${ }^{22} \mathrm{Mcl} 1$ floxed, ${ }^{9} \mathrm{Bcl} x$ floxed ${ }^{45}$ and $\mathrm{Pf} 4-\mathrm{Cre}{ }^{23}$ mice have been previously described. All mouse lines had been backcrossed onto the C57BL/6 background for at least 10 generations prior to this study. Mice were 7-12-weeks old or as otherwise stated. All animal experiments complied with the regulatory standards of, and were approved by, the Walter and Eliza Hall Institute (WEHI) Animal Ethics Committee.

Materials. Dimethyl sulfoxide (DMSO) and propidium iodide were from SigmaAldrich, St. Louis, MO, USA. ABT- $737^{7}$ was provided by Abbott Laboratories, Abbott Park, IL, USA. ABT-199 ${ }^{15}$ and A-1155463.7 $7^{27}$ were provided by AbbVie, North Chicago, IL, USA. The enhanced chemiluminescence system was from Merck Millipore (Kilsyth, VIC, Australia), the protease inhibitor cocktail, Complete, was from Roche (Basel, Switzerland), 4-12\% Bis-Tris gels (NuPAGE) were from Invitrogen Life technologies (Carlsbad, CA, USA), and calibration beads 3.5-4.0 $\mu \mathrm{m}$ were from Spherotech Inc (Lake Forest, IL, USA). Antibodies used for immunoblotting included: mouse anti-BCL-2 (Clone 7; recognises mouse, rat, chicken, dog and human) from BD Transduction Laboratories (Franklin Lakes, NY, USA), rat antiMCL-1 (clone 19C4-15; recognises mouse and human, WEHI mAb Facility, Parkville, VIC, Australia) and anti-actin-HRP from Santa Cruz Biotechnology (Dallas, TX, USA). Antibodies used for flow cytometry included: X488 from Emfret Analytics $\mathrm{GmbH}$ and Co. KG (Eibelstadt, Germany); fluorescently conjugated anti-mouse CD41 (MWReg30) from BD. Anti-platelet serum was purchased from Cedarlane (Burlington, Ontario, Canada).

Haematology. Automated cell counts were performed on blood collected by cardiac puncture or from the retro-orbital plexus into Microtainer tubes containing EDTA (Sarstedt, Ingle Farm, SA, Australia), using an Advia 2120 haematological analyser (Siemens, Munich, Germany). Megakaryocytes were counted manually in sections of sternum and spleen stained with haematoxylin and eosin (H\&E) with a minimum of 10 high-power fields $(\times 200)$ analysed. Images were acquired on a Nikon Eclipse E600 microscope equipped with AxioCam MRc5 (Zeiss, Oberkochen, Germany) and AxioVision 4.8. Scale bars were inserted with Image J. Acute thrombocytopaenia was induced by injection of anti-platelet serum and assessed as described. ${ }^{46}$

Primary megakaryocyte culture. Foetal livers were removed at embryonic day $(E) 13.5$ and transferred into Dulbecco's modified Eagle's medium (high glucose version) with $10 \%$ foetal calf serum (Gibco, Paisley, UK). Bone marrow was flushed into Dulbecco's modified Eagle's medium with $2 \%$ foetal calf serum. The cells were lineage-depleted by incubation with a mix of biotinylated antibodies (CD4, CD2, CD3, CD5, CD8, Ter119, B220, CD19, Gr-1, Ly6G, F4/F8, CD127; WEHI mAb Facility) in KDS-BSS $2 \%$ foetal calf serum, followed by anti-biotin magnetic microbeads (Miltenyi Biotec, Bergisch Gladbach, Germany) and MAC LS columns (Miltenyi Biotec) in EDTA-KDS-BSS $0.5 \%$ foetal calf serum. Single cell suspensions were cultured for 3-5 days at $5 \times 10^{5}$ cells per $\mathrm{ml}$ in serum-free medium ${ }^{47}$ supplemented with $100 \mathrm{ng} / \mathrm{ml}$ murine thrombopoietin (WEHI) at $37^{\circ} \mathrm{C}, 5 \% \mathrm{CO}_{2}$, and mature megakaryocytes purified using a BSA gradient as described.

Megakaryocyte ploidy. Bone marrow was harvested from femora of 8-10week-old mice into $1 \mathrm{ml}$ of CATCH medium and megakaryocyte ploidy was studied by staining with CD41-FITC mAb and propidium iodide, as described. ${ }^{5}$

Caspase activity assay. BSA gradient-purified megakaryocytes $\left(3 \times 10^{4}\right.$ cells per $\mathrm{ml}$ in serum-free medium with TPO) were seeded into 96 -well plates and then incubated at $37^{\circ} \mathrm{C}, 5 \% \mathrm{CO}_{2}$ with or without the addition of ABT-737 (5 $\left.\mu \mathrm{M}\right)$, ABT-199 
$(2.5 \mu \mathrm{M})$ or $\mathrm{A}-1155463.7(0.01-5 \mu \mathrm{M})$. Caspase-Glo $3 / 7$ reagents (Promega, Madison, WI, USA) were added to megakaryocytes after $5 \mathrm{~h}$ of treatment. The luminescence of each sample was determined in a plate-reading LumiSTAR Galaxy luminometer (BMG Labtech, Ortenberg, Germany).

Platelet preparation. Murine blood was obtained by cardiac puncture into 0.1 volume of Aster-Jandl anticoagulant ( $85 \mathrm{mM}$ sodium citrate, $69 \mathrm{mM}$ citric acid, and $20 \mathrm{mg} / \mathrm{ml}$ glucose, $\mathrm{pH} 4.6) .{ }^{48}$ Platelet-rich plasma was obtained by centrifugation at $125 \times g$ for $8 \mathrm{~min}$, followed by centrifugation of the supernatant buffy coat at $125 \times \mathrm{g}$ for $8 \mathrm{~min}$. Platelets were washed by two sequential centrifugations at $860 \times \mathrm{g}$ for $5 \mathrm{~min}$ in $140 \mathrm{mM} \mathrm{NaCl}, 5 \mathrm{mM} \mathrm{KCl}, 12 \mathrm{mM}$ trisodium citrate, $10 \mathrm{mM}$ glucose and $12.5 \mathrm{mM}$ sucrose, $\mathrm{pH} 6.0$ (buffer A). The platelet pellet was resuspended in $10 \mathrm{mM}$ Hepes, $140 \mathrm{Mm} \mathrm{NaCl}, 3 \mathrm{mM} \mathrm{KCl}, 0.5 \mathrm{mM} \mathrm{MgCl}, 10 \mathrm{mM}$ glucose and $0.5 \mathrm{mM}$ $\mathrm{NaHCO}_{3}$, pH 7.4 (buffer B).

Platelet turnover studies. Mice were injected i.v. with $0.15 \mu \mathrm{g} / \mathrm{g}$ body weight of X488 (Emfret), a rat-derived IgG against the platelet CD42c (GPIb $\beta$ ) receptor, and platelet lifespan was measured as previously described. ${ }^{48}$ Platelets were identified in Platelet-rich plasma as being $\mathrm{CD} 41^{+}$by flow cytometry and the proportion of $\mathrm{X}_{488^{+}}$platelets remaining at each time point was assessed.

SDS-PAGE and western blot analysis. Platelets were lysed in NP40 lysis buffer and megakaryocytes were lysed in RIPA buffer. Proteins were separated on 4$12 \%$ Bis-Tris gels (NuPAGE; Invitrogen) under reducing conditions, transferred onto Immobilon-P membranes (Micron Separation), and immunoblotted with various Abs (see above), followed by incubation with secondary HRP-conjugated Abs and ECL.

Platelet counts in newborn mice. Newborn (day P2) mice were given paw tattoos to assist identification. Newborn mice were weighed on P5, 7 and 10 prior to blood collection. Genotyping was performed by PCR using DNA obtained on P2. For blood collection, the anterior facial vein was punctured using a 30-gauge needle. Five microlitres of blood were collected using a micropipette and dispensed into an EDTA blood tube (Sarstedt) with PBS to a total volume of $200 \mu$ l. Automated cell counts were performed on blood collected from newborn mice using an Advia 2120 haematological analyser (Siemens). Reticulated platelets in newborn mice were enumerated using staining with thiazole orange. ${ }^{48}$

In vivo administration of $\mathrm{BH} 3$ mimetics. Mice were treated via oral gavage with $100 \mathrm{mg} / \mathrm{kg} \mathrm{ABT-199}$ administered as a single dose. A stock solution of ABT-199 $(10 \mathrm{mg} / \mathrm{ml}$ ) was diluted in $60 \%$ phosal 50 PG (standardised phosphatidylcholine concentrate with at least $50 \%$ PC and propylene glycol; Phospholipid $\mathrm{GmbH}$, Cologne, Germany), 30\% polyethylene glycol 400 and 10\% ethanol. Blood and sterna were collected at 6 and $24 \mathrm{~h}$ after treatment. Alternatively, mice were injected i.p. with $5 \mathrm{mg} / \mathrm{kg} \mathrm{A}$ - 1155463.7 administered as a single dose. A stock solution of A-1155463.7 (12.5 mg/ml) in DMSO was diluted by addition of $30 \%$ final volume of dosing solution Cremophor ELP:ethanol (ratio 2:1) (Cremophor ELP Sigma-Aldrich) and $5 \%$ dextrose in $\mathrm{H}_{2} \mathrm{O}$ (to reach final volume). Blood, sterna and spleens were collected at 2 and $24 \mathrm{~h}$ after treatment. Sternum and spleen sections were stained with H\&E.

Statistical analyses. Statistical significance between two treatment groups was analysed using an unpaired Student's $t$ test with two-tailed $P$ values. One-way ANOVA with the Bonferroni multiple comparison test was applied where appropriate (GraphPad Prism Software Version 6.0b, La Jolla, CA, USA). ${ }^{*} P<0.05 ;{ }^{* *} P<0.005$; ${ }^{* \star \star} P<0.001$ or as otherwise stated. Data are presented as mean \pm S.D.

\section{Conflict of Interest}

The authors declare no conflict of interest.

Acknowledgements. We thank M. Carpinelli, S. Grabow, J. McManus, S. Green, K. Franks, E. Simankowicz, K. Stoev, M. Do and L. Wilkins for outstanding assistance; and J. Zhang, D. Segal and M. Van Delft for methodological advice. This work was supported by Project Grants (575535, 1079250, 1060179), Program Grants (1016647, 1016701), Fellowships (BTK 1063008, WSA 1058344, AS 1020363) and an Independent Research Institutes Infrastructure Support Scheme Grant (361646) from the Australian National Health and Medical Research Council; a fellowship from the Sylvia and Charles Viertel Foundation (BTK); a fellowship from the German
Research Foundation (IP DFG, PL707/1-1); the Australian Cancer Research Fund, the Science and Industry Endowment Fund; the Sir Edward Dunlop Medical Research Foundation Grant (APN), the Leukemia and Lymphoma Society Specialized Center of Research Grant (7001-13) and a Victorian State Government Operational Infrastructure Support Grant.

\section{Author contributions}

MAD, IP, BTK and ECJ designed research, analysed data and wrote the paper. MAD, IP, RML, ML, JC, MH, APN, CJV and ECJ performed research, analysed data and revised the manuscript. AS, PB, WSA and MS provided reagents, designed research and revised the manuscript.

1. Leslie M. Cell biology. Beyond clotting: the powers of platelets. Science (New York, NY) 2010; 328: 562-564

2. Machlus KR, Italiano JE Jr. The incredible journey: From megakaryocyte development to platelet formation. J Cell Biol 2013; 201: 785-796.

3. Kile BT. The role of apoptosis in megakaryocytes and platelets. Br J Haematol 2014; 165 : 217-226.

4. Wagner KU, Claudio E, Rucker EB 3rd, Riedlinger G, Broussard C, Schwartzberg PL et al. Conditional deletion of the $\mathrm{Bcl}-\mathrm{x}$ gene from erythroid cells results in hemolytic anemia and profound splenomegaly. Development 2000; 127: 4949-4958.

5. Josefsson EC, James C, Henley KJ, Debrincat MA, Rogers KL, Dowling MR et al. Megakaryocytes possess a functional intrinsic apoptosis pathway that must be restrained to survive and produce platelets. J Exp Med 2011; 208: 2017-2031.

6. Kodama T, Takehara T, Hikita H, Shimizu S, Li W, Miyagi T et al. Thrombocytopenia exacerbates cholestasis-induced liver fibrosis in mice. Gastroenterology 2010; 138: 2487-2498, 2498 e2481-2487.

7. Oltersdorf T, Elmore SW, Shoemaker AR, Armstrong RC, Augeri DJ, Belli BA et al. An inhibitor of Bcl-2 family proteins induces regression of solid tumours. Nature 2005; 435: 677-681.

8. Tse C, Shoemaker AR, Adickes J, Anderson MG, Chen J, Jin S et al. ABT-263: a potent and orally bioavailable Bcl-2 family inhibitor. Cancer Res 2008; 68: 3421-3428.

9. Mason KD, Carpinelli MR, Fletcher JI, Collinge JE, Hilton AA, Ellis $S$ et al. Programmed anuclear cell death delimits platelet life span. Cell 2007; 128: 1173-1186.

10. Schoenwaelder SM, Yuan Y, Josefsson EC, White MJ, Yao Y, Mason KD et al. Two distinct pathways regulate platelet phosphatidylserine exposure and procoagulant function. Blood 2009; 114: 663-666.

11. Zhang H, Nimmer PM, Tahir SK, Chen J, Fryer RM, Hahn KR et al. Bcl-2 family proteins are essential for platelet survival. Cell Death Differ 2007; 14: 943-951.

12. Gandhi L, Camidge DR, Ribeiro de Oliveira M, Bonomi P, Gandara D, Khaira D et al. Phase I study of Navitoclax (ABT-263), a novel Bcl-2 family inhibitor, in patients with small-cell lung cancer and other solid tumors. J Clin Oncol 2011; 29: 909-916.

13. Roberts AW, Seymour JF, Brown JR, Wierda WG, Kipps TJ, Khaw SL et al. Substantial susceptibility of chronic lymphocytic leukemia to BCL2 inhibition: results of a phase I study of navitoclax in patients with relapsed or refractory disease. J Clin Oncol 2012; 30: 488-496.

14. Wilson WH, O'Connor OA, Czuczman MS, LaCasce AS, Gerecitano JF, Leonard JP et al. Navitoclax, a targeted high-affinity inhibitor of BCL-2, in lymphoid malignancies: a phase 1 dose-escalation study of safety, pharmacokinetics, pharmacodynamics, and antitumour activity. Lancet Oncol 2010; 11: 1149-1159.

15. Souers AJ, Leverson JD, Boghaert ER, Ackler SL, Catron ND, Chen J et al. ABT-199, a potent and selective $\mathrm{BCL}-2$ inhibitor, achieves antitumor activity while sparing platelets. Nat Med 2013; 19: 202-208.

16. Vandenberg CJ, Cory S. ABT-199, a new Bcl-2-specific BH3 mimetic, has in vivo efficacy against aggressive Myc-driven mouse lymphomas without provoking thrombocytopenia. Blood 2013; 121: 2285-2288.

17. Debrincat MA, Josefsson EC, James C, Henley KJ, Ellis S, Lebois M et al. Mcl-1 and Bcl-x(L) coordinately regulate megakaryocyte survival. Blood 2012; 119: 5850-5858.

18. Kodama T, Hikita H, Kawaguchi T, Shigekawa M, Shimizu S, Hayashi Y et al. Mcl-1 and Bcl$\mathrm{xL}$ regulate $\mathrm{Bak} / \mathrm{Bax}$-dependent apoptosis of the megakaryocytic lineage at multistages. Cell Death Differ 2012; 19: 1856-1869.

19. Liu ZJ, Hoffmeister KM, Hu Z, Mager DE, Ait-Oudhia S, Debrincat MA et al. Expansion of the neonatal platelet mass is achieved via an extension of platelet lifespan. Blood 2014; 123: 3381-3389.

20. Vandenberg CJ, Josefsson EC, Campbell KJ, James C, Lawlor KE, Kile BT et al. Loss of Bak enhances lymphocytosis but does not ameliorate thrombocytopaenia in BCL-2 transgenic mice. Cell Death Differ 2014; 21: 676-684.

21. Roy MJ, Vom A, Czabotar PE, Lessene G. Cell death and the mitochondria: therapeutic targeting of the BCL-2 family-driven pathway. Br J Pharmacol 2014; 171: 1973-1987.

22. Thorp E, Li Y, Bao L, Yao PM, Kuriakose G, Rong J et al. Brief report: increased apoptosis in advanced atherosclerotic lesions of Apoe - / - mice lacking macrophage Bcl-2. Arterioscler Thromb Vasc Biol 2009; 29: 169-172.

23. Tiedt R, Schomber T, Hao-Shen H, Skoda RC. Pf4-Cre transgenic mice allow the generation of lineage-restricted gene knockouts for studying megakaryocyte and platelet function in vivo. Blood 2007; 109: 1503-1506. 
24. Nakayama K, Nakayama K, Negishi I, Kuida K, Sawa H, Loh DY. Targeted disruption of Bcl-2 alpha beta in mice: occurrence of gray hair, polycystic kidney disease, and lymphocytopenia. Proc Natl Acad Sci USA 1994; 91: 3700-3704.

25. Koehler MF, Bergeron P, Choo EF, Lau K, Ndubaku C, Dudley D et al. Structure-guided rescaffolding of selective antagonists of BCL-XL. ACS Med Chem Lett 2014; 5: 662-667.

26. Lessene G, Czabotar PE, Sleebs BE, Zobel K, Lowes KN, Adams JM et al. Structure-guided design of a selective BCL-X(L) inhibitor. Nat Chem Biol 2013; 9: 390-397.

27. Tao ZF, Hasvold L, Wang L, Wang X, Petros AM, Park CH et al. Discovery of a potent and selective BCL-XL inhibitor with in vivo activity. ACS Med Chem Lett 2014; 5: 1088-1093.

28. Brumatti G, Salmanidis M, Kok CH, Bilardi RA, Sandow JJ, Silke N et al. HoxA9 regulated Bcl-2 expression mediates survival of myeloid progenitors and the severity of HoxA9dependent leukemia. Oncotarget 2013; 4: 1933-1947.

29. Ng AP, Kauppi M, Metcalf D, Hyland CD, Josefsson EC, Lebois M et al. Mpl expression on megakaryocytes and platelets is dispensable for thrombopoiesis but essential to prevent myeloproliferation. Proc Natl Acad Sci USA 2014; 111: 5884-5889.

30. Kamada S, Shimono A, Shinto Y, Tsujimura T, Takahashi T, Noda T et al. bcl-2 deficiency in mice leads to pleiotropic abnormalities: accelerated lymphoid cell death in thymus and spleen, polycystic kidney, hair hypopigmentation, and distorted small intestine. Cancer Res 1995; 55: 354-359.

31. Veis DJ, Sorenson CM, Shutter JR, Korsmeyer SJ. Bcl-2-deficient mice demonstrate fulminant lymphoid apoptosis, polycystic kidneys, and hypopigmented hair. Cell 1993; 75: 229-240.

32. Richardson PG, Barlogie B, Berenson J, Singhal S, Jagannath S, Irwin D et al. A phase 2 study of bortezomib in relapsed, refractory myeloma. N Engl J Med 2003; 348: 2609-2617.

33. Montalban-Bravo G, Garcia-Manero G. Novel drugs for older patients with acute myeloid leukemia. Leukemia e-pub ahead of print 21 August 2014.

34. Maxwell SA, Mousavi-Fard S. Non-Hodgkin's B-cell lymphoma: advances in molecular strategies targeting drug resistance. Exp Biol Med (Maywood) 2013; 238: 971-990.

35. Tageja N, Nagi J. Bendamustine: something old, something new. Cancer Chemother Pharmacol 2010; 66: 413-423.

36. Lonial S, Waller EK, Richardson PG, Jagannath S, Orlowski RZ, Giver CR et al. Risk factors and kinetics of thrombocytopenia associated with bortezomib for relapsed, refractory multiple myeloma. Blood 2005; 106: 3777-3784.

37. Shi DS, Smith MC, Campbell RA, Zimmerman PW, Franks ZB, Kraemer BF et al. Proteasome function is required for platelet production. J Clin Invest 2014; 124: 3757-3766.

38. Rudin CM, Hann CL, Garon EB, Ribeiro de Oliveira M, Bonomi PD, Camidge DR et al. Phase II study of single-agent navitoclax (ABT-263) and biomarker correlates in patients with relapsed small cell lung cancer. Clin Cancer Res 2012; 18: 3163-3169.

39. Infante JR, Papadopoulos KP, Bendell JC, Patnaik A, Burris HA 3rd, Rasco D et al. A phase 1b study of trametinib, an oral Mitogen-activated protein kinase kinase (MEK) inhibitor, in combination with gemcitabine in advanced solid tumours. Eur J Cancer 2013; 49 : 2077-2085.

40. Vlahovic G, Karantza V, Wang D, Cosgrove D, Rudersdorf N, Yang J et al. A phase safety and pharmacokinetic study of ABT-263 in combination with carboplatin/paclitaxel in the treatment of patients with solid tumors. Invest New Drugs 2014; 32 976-984.

41. Nakamura S, Takayama N, Hirata S, Seo H, Endo H, Ochi $\mathrm{K}$ et al. Expandable megakaryocyte cell lines enable clinically applicable generation of platelets from human induced pluripotent stem cells. Cell Stem Cell 2014; 14: 535-548.

42. Gras C, Schulze K, Goudeva L, Guzman CA, Blasczyk R, Figueiredo C. HLA-universal platelet transfusions prevent platelet refractoriness in a mouse model. Hum Gene Ther2013; 24: 1018-1028.

43. Lopez JA, Zheng Y. Synthetic microvessels. J Thromb Haemost 2013; 11(Suppl 1): 67-74

44. Thon JN, Mazutis L, Wu S, Sylman JL, Ehrlicher A, Machlus KR et al. Platelet bioreactor-ona-chip. Blood 2014; 124: 1857-1867.

45. Rucker EB 3rd, Dierisseau P, Wagner KU, Garrett L, Wynshaw-Boris A, Flaws JA et al. Bcl-x and Bax regulate mouse primordial germ cell survival and apoptosis during embryogenesis. Mol Endocrinol 2000; 14: 1038-1052.

46. Josefsson EC, Burnett DL, Lebois M, Debrincat MA, White MJ, Henley KJ et al. Platelet production proceeds independently of the intrinsic and extrinsic apoptosis pathways. Nat Comm 2014; 5: 3455.

47. Norol F, Vitrat N, Cramer E, Guichard J, Burstein SA, Vainchenker W et al. Effects of cytokines on platelet production from blood and marrow CD34+ cells. Blood 1998; 91: 830-843.

48. Josefsson EC, White MJ, Dowling MR, Kile BT. Platelet life span and apoptosis. Methods Mol Biol 2012; 788: 59-71.

(i) Cell Death and Disease is an open-access journal published by Nature Publishing Group. This work is licensed under a Creative Commons Attribution 4.0 International License. The images or other third party material in this article are included in the article's Creative Commons license, unless indicated otherwise in the credit line; if the material is not included under the Creative Commons license, users will need to obtain permission from the license holder to reproduce the material. To view a copy of this license, visit http://creativecommons.org/licenses/by/4.0/

Supplementary Information accompanies this paper on Cell Death and Disease website (http://www.nature.com/cddis) 